\title{
EUS-guided biliary drainage is equivalent to ERCP for primary treatment of malignant distal biliary obstruction: a systematic review and meta-analysis
}

\section{(ㄷ)(우우}

\author{
Authors \\ Kelly E. Hathorn 1, ${ }^{*}$, Ahmad Najdat Bazarbashi 1, ${ }^{*}$, Jordan S. Sack1, Thomas R. McCarty1, Thomas J. Wang², Walter W. \\ Chan ${ }^{1}$, Christopher C. Thompson ${ }^{1}$, Marvin Ryou ${ }^{1}$
}

Institutions

1 Division of Gastroenterology, Hepatology and Endoscopy, Brigham and Women's Hospital. Harvard Medical School, Boston, Massachusetts, United States

2 Division of Internal Medicine, Massachusetts General Hospital, Harvard Medical School, Boston, Massachusetts, United States

submitted 21.3.2019

accepted after revision 5.7.2019

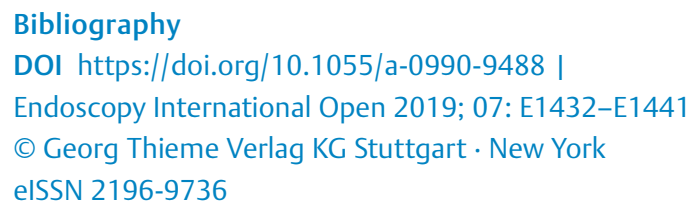

Corresponding author

Marvin Ryou, MD, Division of Gastroenterology, Hepatology and Endoscopy, Brigham and Women's Hospital, Boston, MA 02115, USA

Fax: +1-617-264-6342

mryou@bwh.harvard.edu

Supplemental Figs. 1-9, Supplemental Table Online content viewable at: https://doi.org/10.1055/a-0990-9488

\section{ABSTRACT}

Background and study aims Although endoscopic retrograde cholangiopancreatography (ERCP) is standard of care for malignant biliary obstruction, endoscopic ultrasoundguided biliary drainage (EUS-BD) as a primary treatment has become increasingly utilized. The aim of this study was to perform a systematic review and meta-analysis to evaluate the effectiveness and safety of EUS-BD for primary treatment of malignant biliary obstruction and comparison to traditional ERCP.

Methods Individualized search strategies were developed through November 2018 using PRISMA and MOOSE guidelines. A cumulative meta-analysis was performed by calculating pooled proportions. Subgroup analysis was performed for studies comparing EUS-BD versus ERCP. Heterogeneity was assessed with Cochran $Q$ test or $l^{2}$ statistics, and publication bias by funnel plot and Egger's tests.

Results Seven studies ( $\mathrm{n}=193$ patients; $57.5 \%$ males) evaluating primary EUS-BD for malignant biliary obstruction were included. Mean age was 67.4 years (2.3) followed an average of 5.4 months (1.0). For primary EUS-BD, pooled technical success, clinical success, and adverse event (AE) rates were $95 \%$ (95\% Cl $91-98)$, 97\% (95\% Cl $93-100)$, and $19 \%(95 \% \mathrm{Cl} 11-29)$, respectively. Among EUS-BD and ERCP comparator studies, technical and clinical success, and total AEs were not different with lower rates of post-ERCP pancreatitis and reintervention among the EUSBD group.

Conclusion Primary EUS-BD is an effective treatment with few AE. Comparing EUS-BD versus ERCP, EUS-BD has comparable efficacy and improved safety as a primary treatment for malignant biliary obstruction. Further randomized trials should be performed to identify patient populations and clinical scenarios in which primary EUS-BD would be most appropriate.

\section{Introduction}

Historically, endoscopic retrograde cholangiopancreatography (ERCP) has been the conventional method of therapeutic relief of malignant distal biliary obstruction [1-3]. Although conven-

\footnotetext{
* These authors contributed equally
}

tional ERCP is being considered standard of care, overall adverse event $(A E)$ rates with it (i. e. transpapillary access) have been reported to be as high as $36 \%$ with a procedure failure rate of $3 \%$ to $10 \%$ for malignant biliary obstruction [4-9]. Although interventional radiology (IR)-guided percutaneous transhepatic biliary drainage may be an alternative to complicated or failed ERCP, this more invasive option carries increased 
risk of pain at the insertion site, potential for long-standing external biliary drainage, and has been associated with an increased higher risk of bacteremia, cholangitis, or hemobilia [1, $10-13]$

Given the significant AE rate associated with traditional ERCP and limitations of the IR-guided percutaneous biliary approach, endoscopic ultrasound-guided biliary drainage (EUS-BD) has become increasingly utilized over the last two decades. Originally described by Giovannini in 2001, EUS-BD provided an alternative to achieve successful biliary drainage after failed ERCP [14]. Since this first procedure, EUS-BD has come to the forefront of therapy for biliary obstruction, particularly as a rescue procedure in cases where standard endoscopic or percutaneous measures are not feasible or have been unsuccessful [15-34]. While multiple retrospective and prospective studies have identified rescue EUS-BD as a technically feasible and clinically successful second-line procedure, few studies have evaluated its effectiveness as a primary treatment for malignant distal biliary obstruction [34].

Allowing for direct visualization and access to the biliary tree through sonographic guidance, EUS-BD may have several benefits over traditional ERCP and may decrease the significant risk of post-ERCP pancreatitis [7, 10,35] Given the limited data on EUS-BD as primary modality for biliary drainage, the primary aim of this study was to perform a structured systematic review and meta-analysis to investigate pooled technical success, clinical success, and AE rates for primary EUS-BD in malignant distal biliary obstruction. Secondary aims of this study were to evaluate comparative effectiveness and safety of primary EUS$\mathrm{BD}$ as compared to traditional ERCP.

\section{Patients and methods}

\section{Study design and search strategy}

This systematic review was performed in accordance with the Preferred Reporting Items for Systematic Reviews and MetaAnalyses (PRISMA) statement outline for reporting systematic reviews and meta-analyses and was conducted following a priori established protocol [36]. Given inclusion of observation studies, Meta-Analysis of Observational Studies in Epidemiology (MOOSE) reporting guidelines were also followed [37].

Individualized search strategies for PubMed, Embase, Cochrane Central Register of Controlled Trials and U.S. National Library of Medicine's (NLM) clinical trials were performed from inception through November 30, 2018. For PubMed and Embase searches, medical subject heading (MESH) and Emtree terms as well as title/abstract keyword search terms were used in combination: cholestasis, bile duct, biliary, obstruction, drainage, endosonography, endoscopic ultrasound, EUS, EUSbiliary drainage (EUS-BD), EUS-anterograde cholangiopancreatography (EACP). For Cochrane Central Register of Controlled Trials, we performed a search using cholestasis [MeSH] and EUS [search item]. We also searched NLM's clinical trials for completed studies involving biliary obstruction and endoscopic ultrasound as search terms. An experienced medical librarian at Harvard Medical School assisted with our search strategy.
All relevant articles irrespective of year of publication, type of publication, or publication status were included. Titles and abstracts of all potentially relevant studies were screened for eligibility. Duplicates were removed by the Covidence Systematic Review Software (Veritas Health Innovation, Melbourne, Australia). Reference lists of studies of interest were then manually reviewed for additional articles by cross-checking bibliographies. Two reviewers (KEH and ANB) independently screened titles and abstracts of all the articles according to predefined inclusion and exclusion criteria. Any differences were resolved by mutual agreement and in consultation with the third reviewer (JSS). For studies with incomplete information, contact was attempted with the principal authors to obtain additional data to ascertain eligibility.

\section{Selection criteria}

Eligibility criteria were predetermined and included adult patients aged $\geq 18$ years with malignant distal biliary obstruction. Only studies investigating use of EUS-BD (utilizing transluminal or transpapillary, including rendezvous techniques) for primary management of malignant distal biliary obstruction were included. EUS-BD for benign biliary obstruction or procedure performed in individuals with surgically altered anatomy without underlying malignancy were also excluded. Studies involving alternative endoscopic, medical, surgical modalities, or studies evaluating EUS-BD as a rescue or second-line therapy were excluded. Studies were required to report the primary aim of technical or clinical success and AE rate to be included. Randomized controlled trials, observational studies, and case series were included. Multiple published work from similar authors was evaluated independently by three reviewers (KEH, ANB, JSS) for overlapping enrollment times to preserve independence of observations. Authors were contacted via email to ensure the procedure was primary EUS therapy as well as to further clarify any concerns for overlapping enrollment times after manuscript review. A study was excluded if deemed to have insufficient data, as were review articles, editorials, and correspondence letters that did not report independent data. Case series and reported studies with fewer than 10 patients were excluded.

\section{Measured outcomes}

The primary outcome of this study was effectiveness and safety of EUS-BD for primary treatment of malignant biliary obstruction as determined by technical success, clinical success, and AEs. Along with baseline study and patient characteristics, additional measured outcomes, when available, included rate of reintervention for EUS-BD as well as similar ERCP-related outcomes for comparator studies.

\section{Risk of bias and quality assessment}

Risk of bias was assessed using the Cochrane Collaboration's risk of bias in non-randomized studies of interventions (ROBINS-I) tool for observational studies and the Cochrane Collaboration's tool for assessing risk of bias for randomized clinical trials (RCTs). In this study, publications were deemed low risk of bias if $\geq 50 \%$ of the above domains were judged as low risk. 
Quality of observational studies was evaluated using the Newcastle-Ottawa Quality Assessment Scale. Domains assessed were selection of cohort, ascertainment of exposure/comparator, and assessment of outcome. Quality of RCTs was assessed using the JADAD score. In this study, high quality was defined as a Newcastle-Ottawa Quality Assessment Scale score $\geq 6$ or a JADAD score $\geq 3$. Two authors (KEH and ANB) independently extracted data and assessed risk of bias and study quality for each of the articles. Any disagreements were resolved by discussion and consensus, with the third author (JSS) serving as the final arbiter if consensus could not be reached.

\section{Investigations of heterogeneity}

Heterogeneity was assessed for the individual meta-analyses using the chi-squared test and the $I^{2}$ statistic. Significant heterogeneity was defined as $P<0.05$ using the Cochran $Q$ test or $R^{2}>50 \%$, with values $>50 \%$ indicating substantial heterogeneity [38]. Further quantification of heterogeneity was categorized based upon $R^{2}$ with values of $25 \%, 50 \%$, and $75 \%$ indicating low, moderate, and high amounts of heterogeneity, respectively. Given use of the random effects model to estimate average effect, a $95 \%$ prediction interval was calculated to determine dispersion of effects and clearly illustrate heterogeneity in the calculated effect size [39].

\section{Assessment of publication bias}

With regard to publication bias, funnel plot asymmetry and Egger's regression test were performed as well [40]. Differences in subgroups were assessed using the Cochran $Q$ test for interaction with a $P<0.05$ defined as statistically significant. The trim and fill method was used to correct for funnel plot asymmetry and provide an adjusted effect. The classic fail-safe test was also applied to assess risk of bias across studies.

\section{Statistical analyses}

This systematic review was performed by means of proportion meta-analysis. After appropriate studies were identified through systematic review, the individual study proportion was transformed into a quantity using the Freeman - Tukey variant of the arcsine square root transformed proportion. Then the pooled proportion was calculated as the back transform of the weighted mean of the transformed proportions, DerSimonian-Laird weights for the random effects model [41-43]. The pooled rates were estimated using random effects models and presented as point estimates (rates) with $95 \%$ confidence intervals. In contrast to fixed effect models, which are used to estimate a common effect, random effect models estimate an average effect, and the variability of the effects represented by their average may have clinical implications [39].

Sensitivity analyses were performed to include only data included in RCTs as determined via PRISMA guidelines, excluding observational studies. In addition, data for observation studies only, as reported by MOOSE guidelines and data limited to only high-quality studies were also reported. Subgroup analyses were also performed to compare EUS-BD versus ERCP. For this, only studies including both EUS-BD and ERCP data were consid- ered. We used tabular and graphical displays in Review Manager 5 (RevMan 5.3) for subgroup analysis to compare EUS-BD to ERCP. Combined weighted proportions and additional analyses were determined by use of the Stata 13.0 software package (Stata Corp LP, College Station, Texas, United States). $P<0.05$ was defined as statistically significant. Continuous variables were presented as mean (standard deviation), and differences between groups were tested using the $t$-test.

\section{Results}

\section{Characteristics of included studies}

This systematic review and meta-analysis included a total of seven studies $(n=193)[10,11,44-48]$ A PRISMA flow chart of search results is shown in $\mathbf{F i g}$. $\mathbf{1}$. Three RCTs, three prospective studies, and one retrospective cohort study were included. Mean age of patients was 67.4 years (2.3), $57.5 \%$ males, with an average study follow-up period of 5.4 months (1.0). All patients had malignant biliary obstruction with a mean common bile duct dilation of $13.76 \mathrm{~mm}$ (1.73). Mean EUS-BD procedure duration was 27.8 minutes (5.8). Characteristics of all included studies are illustrated in $>$ Table 1.

\section{Cumulative clinical effectiveness and safety}

Overall, primary EUS-BD was effective and safe with results listed in $>$ Table 2 . The pooled technical success rate and clinical success rate for EUS-BD was $95 \%$ [(95\% Cl 91 to 98$) ; R=$ $00.00 \%$; prediction interval 0.87 to 0.97 , range 0.10 ] and $97 \%$ [( $95 \% \mathrm{Cl} 93$ to 100$) ; R^{2}=16.70 \%$; prediction interval 0.86 to 0.97 , range 0.11 ], respectively ( $\boldsymbol{F}$ Fig. $\mathbf{2 a}$ and $\boldsymbol{\nabla}$ Fig. $\mathbf{2 b}$ ). The pooled AE rate was $19 \%$ [( $95 \% \mathrm{Cl} 11$ to 29$) ; l^{2}=56.00 \%$; prediction interval -0.89 to 0.95 , range 1.84 ] with the most frequent complications being bile peritonitis and cholangitis ( $\mathbf{F i g . 2 c}$ ). Rate of reintervention was $7 \%$ [(95\% Cl 2 to 13$) ; R^{2}=41.49 \%$; prediction interval 0.03 to 0.20 , range 0.17$]$ ( $\triangleright$ Fig. 2 d).

\section{Sensitivity analyses}

Excluding low-quality studies revealed all included RCTs, with a pooled technical success rate, clinical success rate, $A E$ rate, and rate of reintervention of [ $93 \%(95 \% \mathrm{Cl} 87$ to 98$\left.) ; I^{2}=00.00 \%\right)$; $95 \%$ (95\% Cl 88 to 99$\left.) ; R^{2}=17.46 \%\right) ; 14 \%(95 \% \mathrm{Cl} 8$ to 21$) ; R^{2}=$ $00.00 \%)$; and $6 \%(95 \% \mathrm{Cl} 0$ to 18$\left.) ; R^{2}=66.62 \%\right)$, respectively (Supplemental Fig.1a-d) $[10,11,44]$. RCT data were the same as high-quality-only data. Observational studies demonstrated pooled technical success rate, clinical success rate, adverse event rate, and rate of reintervention was similar [ $97 \%$ (95\% Cl 91 to 100); $\left.R^{2}=00.00 \%\right) ; 99 \%(95 \% \mathrm{Cl} 94$ to 100$) ; R^{2}=$ $8.65 \%) ; 24 \%(95 \% \mathrm{Cl} 9$ to 44$\left.) ; R^{2}=69.24 \%\right)$; and $7 \%(95 \% \mathrm{Cl} 1$ to 17$) ; P^{2}=21.70 \%$ ), respectively] (Supplemental Fig. $2 \mathrm{a}-\mathbf{d}$ ).

\section{Subgroup analyses}

Five studies directly compared EUS-BD versus ERCP $[10,11$, $44-46$ ]. Of these studies ( $n=159$ patients in each cohort; total $\mathrm{n}=318$ in both cohorts), EUS-BD patients were older with a mean age was $68.53 \pm 1.19$ years versus $67.38 \pm 2.55$ years $(P<$ 0.001). Furthermore, among EUS-BD and ERCP comparator studies, technical success [OR 1.30 (95\% Cl 0.38 to 4.50$)$; $P=$ 


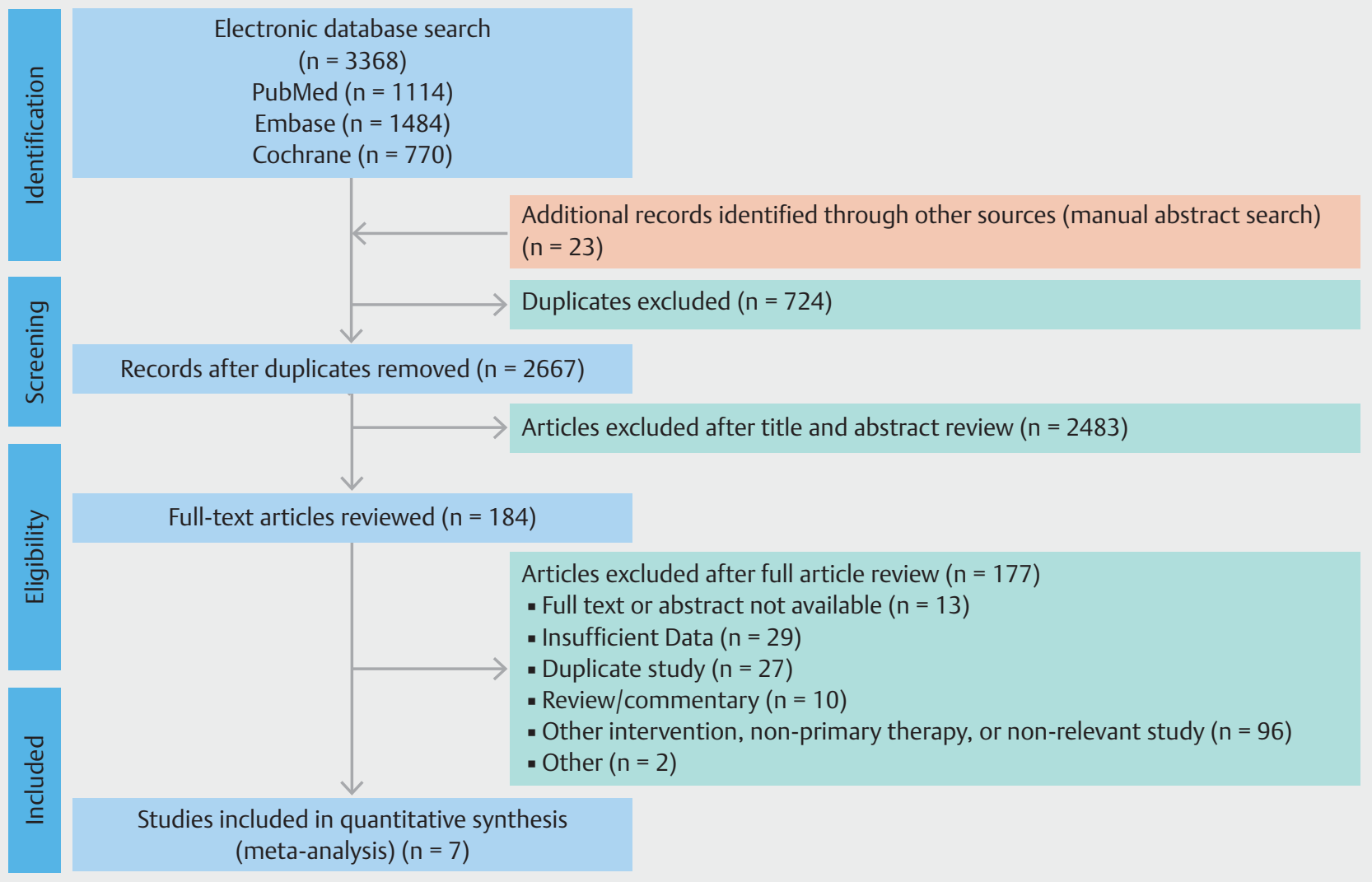

- Fig. 1 Preferred Reporting Items for Systematic Reviews and Meta-Analyses (PRISMA) Flowchart of search results for the EUS-BD for treatment of distal malignant biliary obstruction.

$0.68 ; P^{2}=33.00 \%$ and clinical success [OR $2.32(95 \% \mathrm{Cl} 0.48$ to 11.15) $P=0.29 ; R^{2}=51.00 \%$ ] were not statistically different ( $\triangleright$ Fig. 3 a and $\triangleright$ Fig.3b, respectively). While primary EUS-BD was associated with no difference in rate of total AEs [OR 0.70 (95\% Cl 0.24 to 2.03 ) $P=0.51 ; I^{2}=70.00 \%$ ], rate of post-ERCP pancreatitis was significantly lower for EUS-BD [OR $0.17(95 \%$ $\mathrm{Cl} 0.04$ to 0.79 ) $P=0.02 ; R^{2}=0.00 \%$ ] compared to traditional ERCP ( $\triangleright$ Fig.4a and Supplemental Fig.3, respectively). PostERCP pancreatitis occurred in $6.00 \%$ of patients with no events in the EUS-BD cohort. In addition, EUS-BD resulted in a significantly decreased rate of reintervention as compared to traditional ERCP [OR $0.23(95 \% \mathrm{Cl} 0.10$ to 0.49$) P<0.001 ; R=$ $1.00 \%$ ] ( Fig.4b). Cumulative EUS-BD data and comparative data are shown in $>$ Table 2 . Individual EUS-BD and ERCP study characteristics of clinical success, technical success, and detailed reporting of procedure-associated AEs are presented in Supplemental Table.

\section{Risk of bias assessment}

All seven studies were assessed using GRADE scoring system, which assigned low grade to the three observational studies, and an initial high grade to all three randomized studies. However, one of the randomized studies was downgraded to moderate quality based on further review ( $\triangleright$ Table 1 ). Authors “ judgements about each risk of bias for all included studies is high- lighted in Supplemental Fig. 4. A risk of bias summary graph is also available in Supplemental Fig. 5. For all primary EUS studies comparing EUS-BD to ERCP, there was no evidence of publication bias, based upon either qualitative on funnel-plot asymmetry or quantitative (i.e., Egger regression test, $P>0.05$ ) for technical and clinical success as well as rate of reintervention and total adverse events (Supplemental Figs.6-9, respectively). Funnel plot for comparative studies of EUS-BD versus ERCP for AEs appeared symmetric with two studies as an outlier; however, Egger's test revealed $P>0.05$ indicating no publication bias (Supplemental Fig. 9).

\section{Discussion}

Over the course of the last two decades, EUS-BD has come to the forefront of therapy as a safe and effective treatment for malignant biliary obstruction, particularly in cases where standard endoscopic or percutaneous measures are not feasible or have failed. EUS-BD allows for direct access to the biliary tree through sonographic guidance. Consequently, there are two major approaches or access sites for the procedure, intrahepatic or extrahepatic $[34,35]$. With the intrahepatic technique, the left lobe of the liver is accessed through the gastric wall, and with the extrahepatic technique, the common bile duct is 


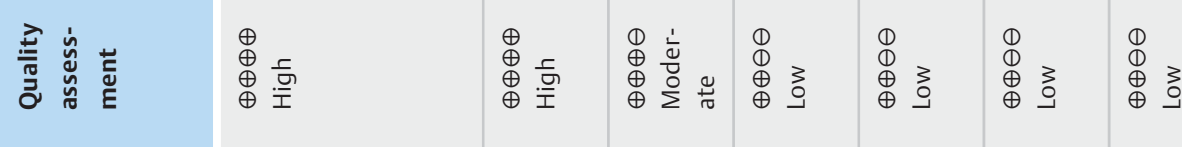

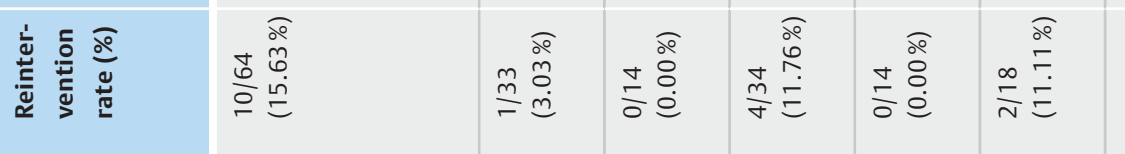

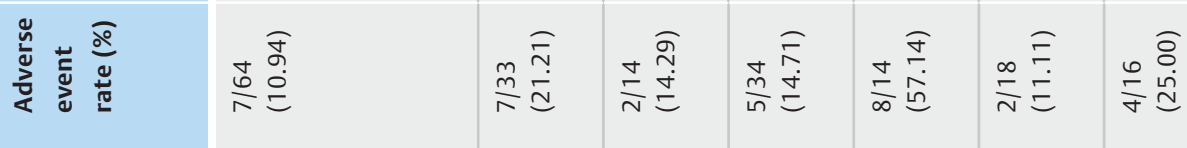

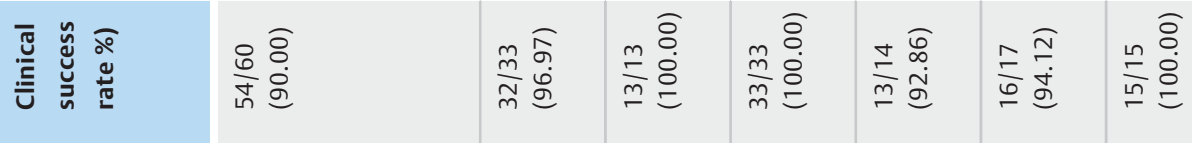

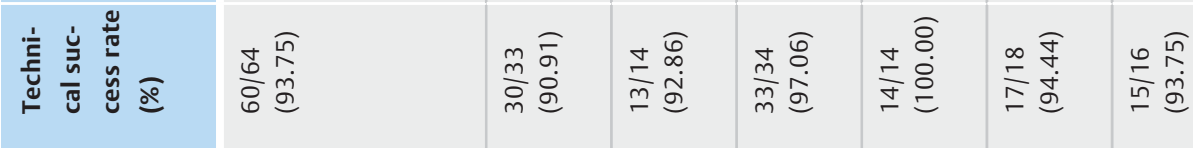

ํํㅁ

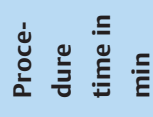

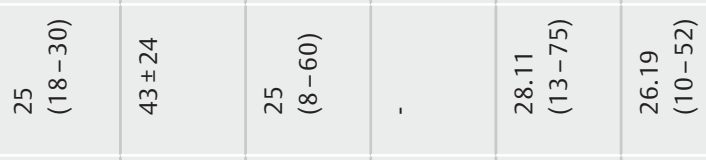

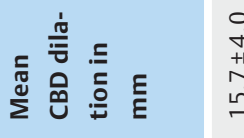

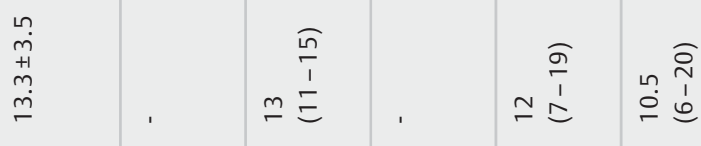

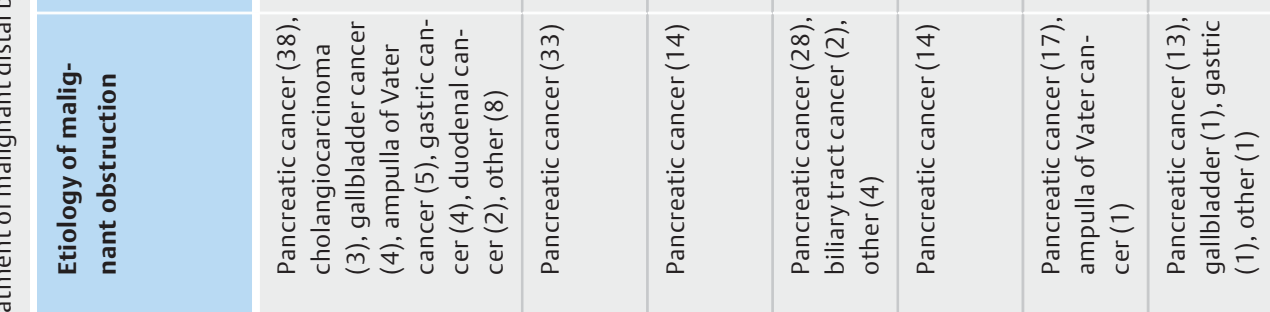

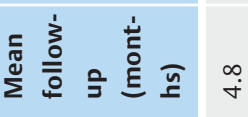

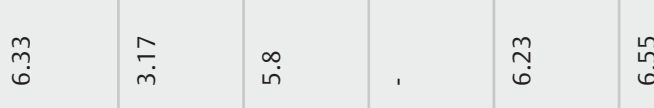

离

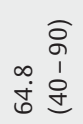

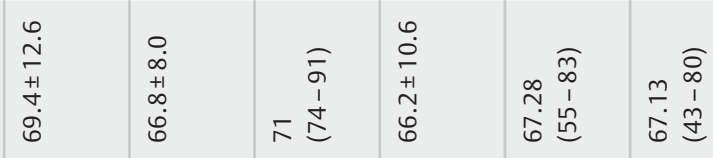

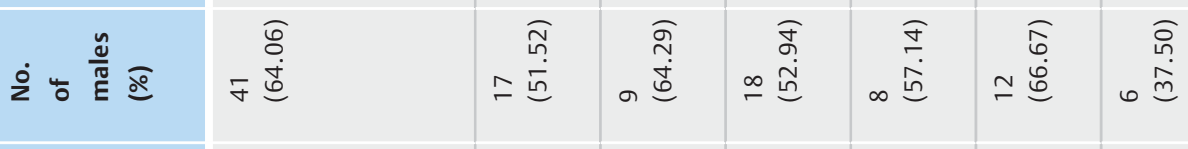

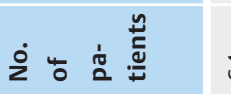

高

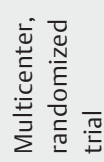

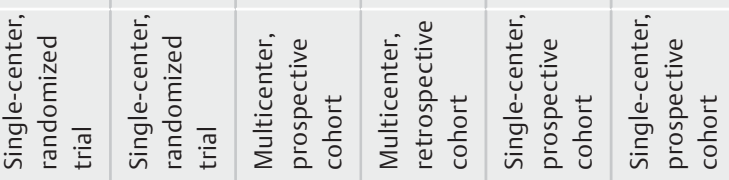

ֻั

$\stackrel{\infty}{\overbrace{\infty}^{\infty}}$

安旁

흘

\begin{tabular}{|c|c|c|c|c|}
\hline$\stackrel{\infty}{\grave{N}}$ & $\stackrel{\infty}{\stackrel{\infty}{N}}$ & $\stackrel{\infty}{\vdots}$ & $\stackrel{m}{i}$ & $\bar{i}$ \\
\hline 高离 & 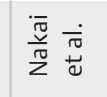 & 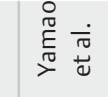 & 离产 & 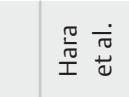 \\
\hline
\end{tabular}




\section{Study}

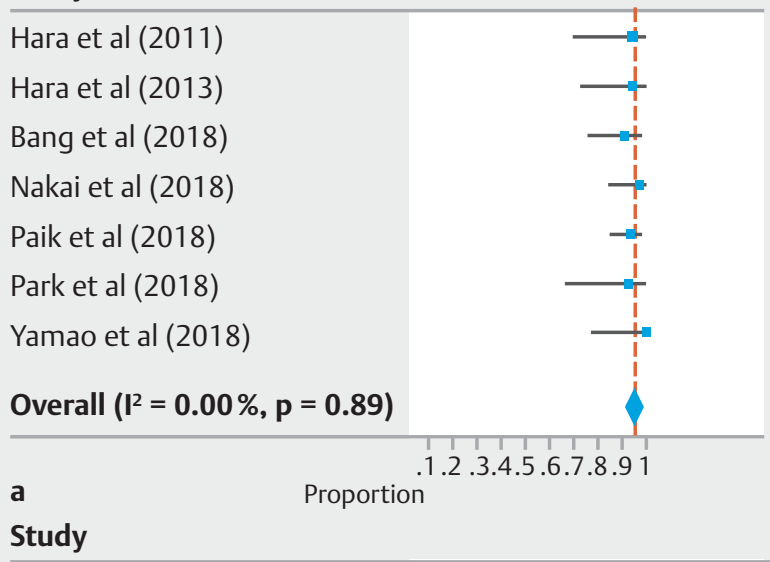

Hara et al (2011)

Hara et al (2013)

Bang et al (2018)

Nakai et al (2018)

Paik et al (2018)

Park et al (2018)

Yamao et al (2018)

Overall $\left(I^{2}=16.70 \%, p=0.30\right)$

b

Proportion

\section{Study}

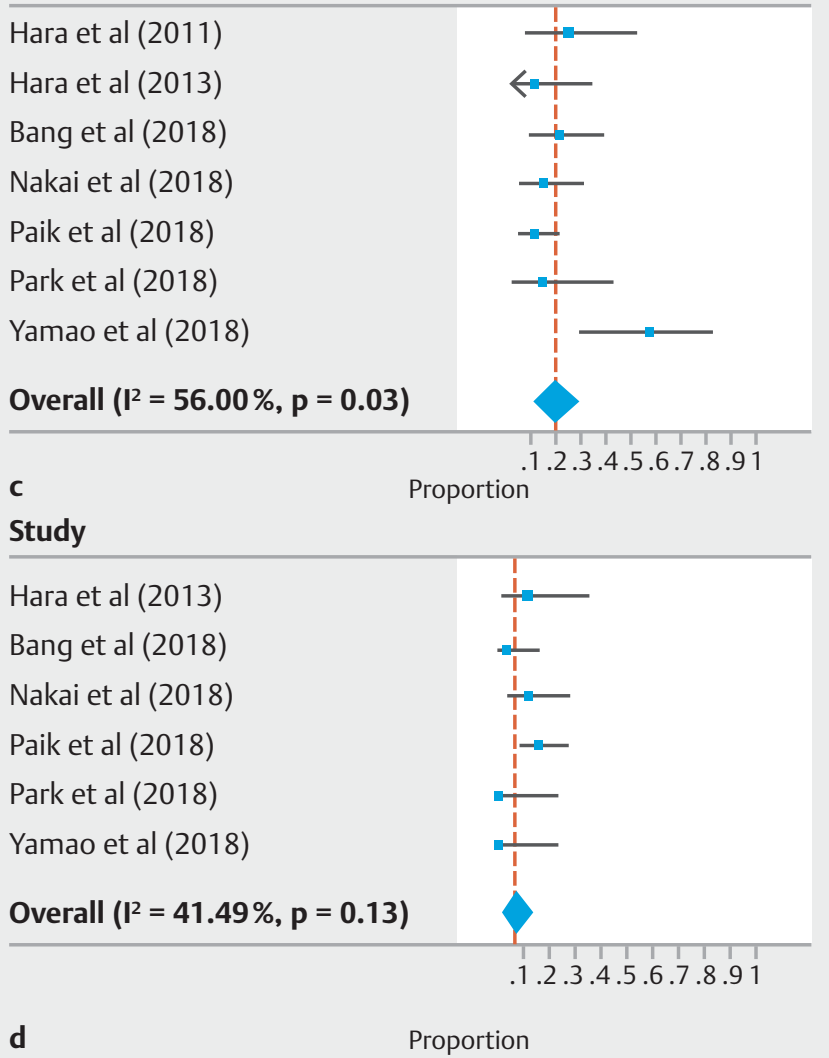

- Fig. 2a Technical success rate for EUS-BD for treatment of distal malignant biliary obstruction. $\mathbf{b}$ Clinical success rate for EUS-BD for treatment of distal malignant biliary obstruction. $\mathbf{c}$ Serious adverse events for EUS-BD for treatment of distal malignant biliary obstruction $\mathbf{d}$ Rate of reintervention for EUS-BD for treatment of distal malignant biliary obstruction.

accessed directly through the duodenum (i. e., choledochoduodenostomy).

Previous studies have shown EUS-BD to be an effective modality as a rescue therapy after failed ERCP. Khan et. al. conducted a meta-analysis, involving 20 studies evaluating EUS-BD in patients with failed ERCP, in which they demonstrated weighted pool rates for technical success and post-procedure-related AEs of $90 \%$ (95\% Cl 86 to 93 ) and $17 \%(95 \% \mathrm{Cl} 13$ to 22$)$, respectively [49]. More recently, Mohan and colleagues conducted a meta-analysis involving 13 studies on EUS-guided choledochoduodenostomy as primary and as rescue therapy, in which they demonstrated a pooled technical success rate of $91.9 \%$ (95\% Cl 88.0 to 94.6$)$, pooled clinical success rate of $91.9 \%$ (95\% Cl 86.6 to 95.2$)$, and $\mathrm{AE}$ rate of $14.5 \%(95 \% \mathrm{Cl}$ 10.7 to 19.3 ) [50]. Thus, given the overall promising results of EUS-BD in cases of failed ERCP, several studies have evaluated its role as primary modality for biliary drainage, irrespective of ERCP candidacy. Yet, despite this more frequent adoption as a primary tool, there remains a paucity of literature available to determine overall effectiveness and safety, as well as comparative analyses to a standard transpapillary ERCP approach.

The results of this meta-analysis confirm that EUS-BD as primary therapy is technically feasible, clinically successful, and relatively safe for treatment of malignant biliary obstruction. We demonstrated pooled technical and clinical success rates of $95 \%$ and $97 \%$, respectively. Need for repeat endoscopic procedure between the two techniques demonstrated EUS-BD to have an $80 \%$ lower rate of reintervention as compared to traditional ERCP. In addition, given the ability for EUS-BD to potentially avoid traumatic papillary manipulation, it offers a theoretically significant advantage clinically or practically over ERCP by reducing risk of post-ERCP pancreatitis [7, 10,35]. Results from this meta-analysis suggest this assumption is true with $87 \%$ lower odds of developing post-procedure pancreatitis with EUS-BD compared to ERCP. Rates of post-ERCP pancreatitis seen in the included studies were similar to those reported in previous literature [51]. It may be inferred this reduction in pancreatitis resulted in a significant number of total events overall; however, at least one RCT demonstrated EUS-BD to have a higher rate of stent patency with less intervention and better preservation of quality of life as compared to ERCP [10]. While this was not shown in our meta-analysis, lower risk of post-procedure pancreatitis, combined with a reduction in need for reintervention and equivalent clinical and technical success rates compared to ERCP, may suggest an even larger 


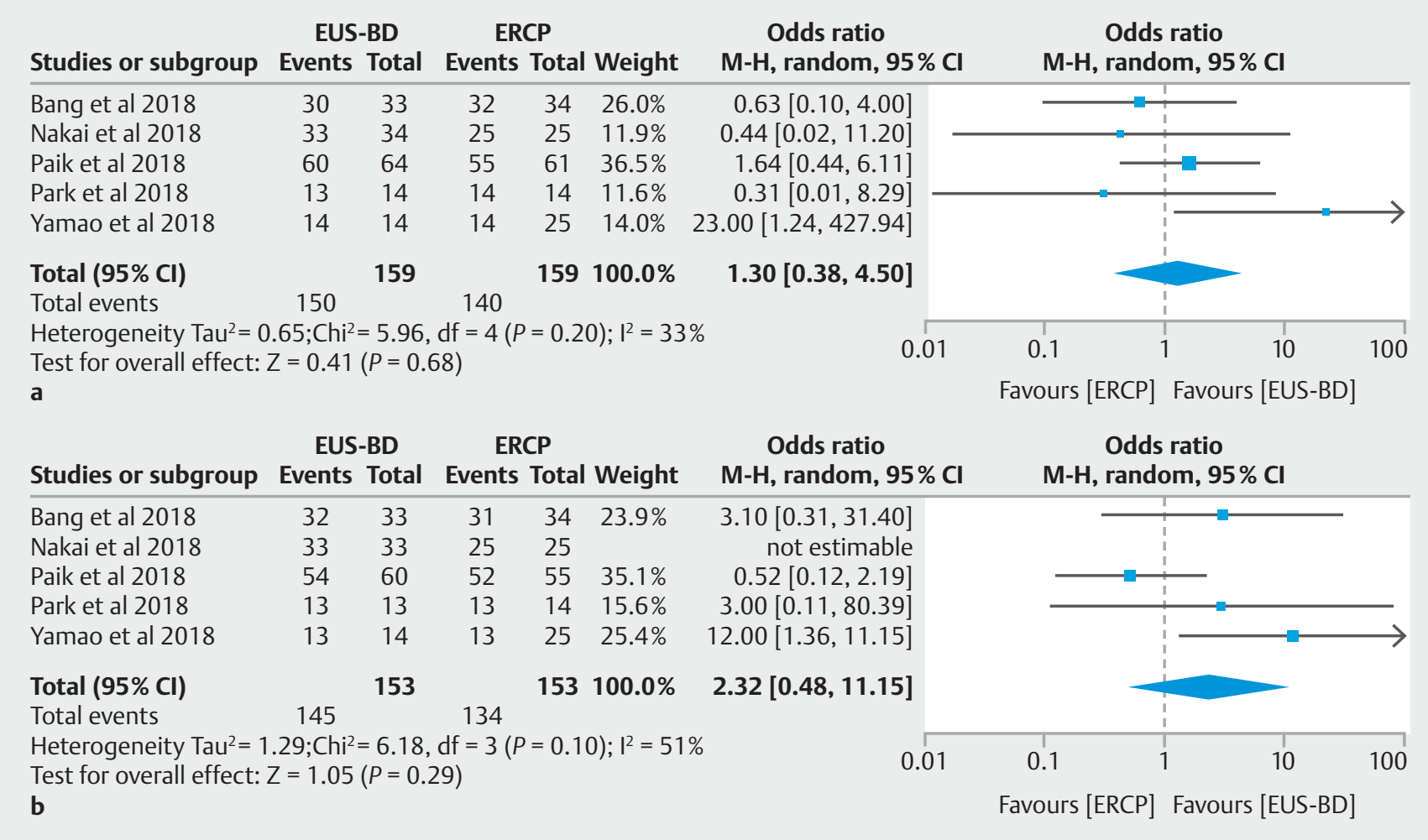

- Fig.3a Comparative technical success rate of EUS-BD versus ERCP for treatment of distal malignant biliary obstruction. $\mathbf{b}$ comparative clinical success rate of EUS-BD versus ERCP for treatment of distal malignant biliary obstruction.

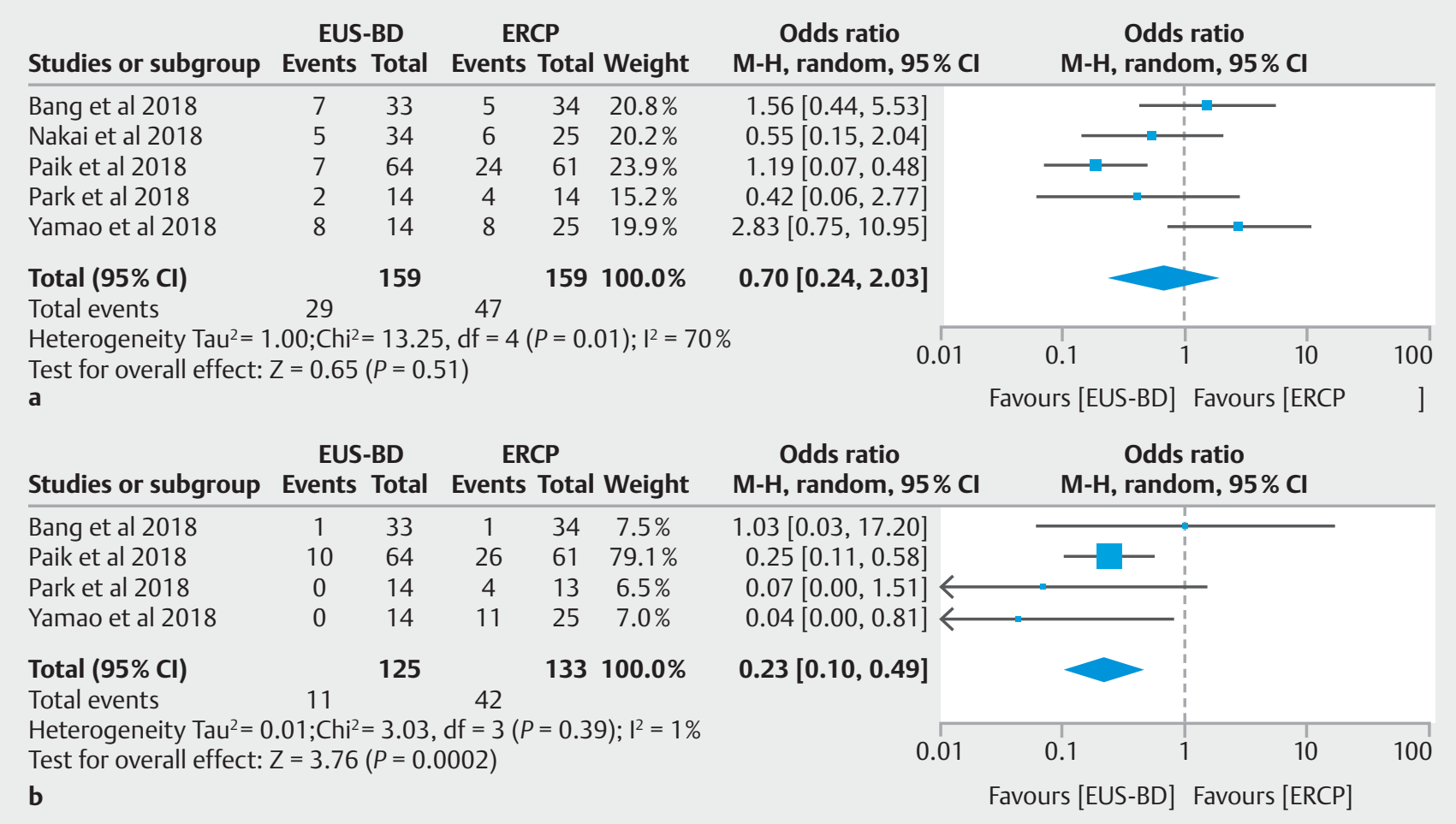

- Fig. 4a Comparative adverse event rate of EUS-BD versus ERCP for treatment of distal malignant biliary obstruction. $\mathbf{b}$ Comparative reintervention rate of EUS-BD versus ERCP for treatment of distal malignant biliary obstruction. 
- Table 2 Efficacy and safety of EUS-BD for malignant biliary obstruction: cumulative and comparative meta-analysis.

\begin{tabular}{|c|c|c|c|c|}
\hline & \multicolumn{2}{|c|}{ Cumulative data for EUS-BD } & \multicolumn{2}{|c|}{ Comparative data for EUS-BD vs ERCP } \\
\hline & Pooled rate $(95 \% \mathrm{Cl})$ & Heterogeneity $\left(I^{2}\right)$ & Odds ratio $(95 \% \mathrm{Cl})$ & Heterogeneity $\left(\mathrm{I}^{2}\right)$ \\
\hline Technical success & $95 \%$ (95\% Cl 91 to 98$)$ & $0.00 \%$ & $1.30(95 \% \mathrm{Cl} 0.38$ to 4.50$)$ & $33.00 \%$ \\
\hline Clinical success & $97 \%$ (95\% Cl 93 to 100$)$ & $16.70 \%$ & $2.32(95 \% \mathrm{Cl} 0.48$ to 11.15$)$ & $51.00 \%$ \\
\hline Total adverse events & $19 \%$ (95\% Cl 11 to 29$)$ & $56.00 \%$ & $0.70(95 \% \mathrm{Cl} 0.24$ to 2.03$)$ & $70.00 \%$ \\
\hline - Pancreatitis & $0 \%(95 \% \mathrm{Cl} 0$ to 1$)$ & $0.00 \%$ & $0.17(95 \% \mathrm{Cl} 0.04$ to 0.79$)$ & $0.00 \%$ \\
\hline - Bile peritonitis & $3 \%(95 \% \mathrm{Cl} 0$ to 8$)$ & $40.19 \%$ & $2.91(95 \% \mathrm{Cl} 0.12$ to 72.70$)$ & $\mathrm{NA}^{*}$ \\
\hline - Pneumoperitoneum & $0 \%(95 \% \mathrm{Cl} 0$ to 2$)$ & $0.00 \%$ & $1.79(95 \% \mathrm{Cl} 0.19$ to 16.66$)$ & $0.00 \%$ \\
\hline - Cholangitis & $2 \%(95 \% \mathrm{Cl} 0$ to 7$)$ & $57.58 \%$ & $0.74(95 \% \mathrm{Cl} 0.33$ to 1.68$)$ & $0.00 \%$ \\
\hline - Cholecystitis & $1 \%(95 \% \mathrm{Cl} 0$ to 5$)$ & $25.51 \%$ & $0.75(95 \% \mathrm{Cl} 0.14$ to 3.90$)$ & $37.00 \%$ \\
\hline - Stent migration & $0 \%(95 \% \mathrm{Cl} 0$ to 2$)$ & $0.00 \%$ & $0.74(95 \% \mathrm{Cl} 0.10$ to 5.37$)$ & $11.00 \%$ \\
\hline - Stent dysfunction/occlusion & $0 \%(95 \% \mathrm{Cl} 0$ to 2$)$ & $5.00 \%$ & $0.30(95 \% \mathrm{Cl} 0.06$ to 1.47$)$ & $0.00 \%$ \\
\hline Reintervention rate & $7 \%(95 \% \mathrm{Cl} 2$ to 13$)$ & $41.49 \%$ & $0.23(95 \% \mathrm{Cl} 0.10$ to 0.49$)$ & $1.00 \%$ \\
\hline
\end{tabular}

role for EUS-BD primary therapy by endoscopists with familiarity with EUS-BD.

Limitations to the current study include the inherent heterogeneity bias of pooled systematic reviews and meta-analyses. While we attempted to assess for this as well as risk of bias, specific limitations include differences in patient population across EUS-BD and ERCP, differences in short-term and longterm follow-up periods, and inclusion of both intrahepatic and extrahepatic EUS-BD access. Based upon study reporting, subgroup analysis by choledocoduodenostomy versus hepaticogastrostomy was not possible. Furthermore, both randomized and non-randomized studies were included in this analysis. Heterogeneity was significant for AEs between studies though low for technical and clinical success rates. Given use of odds ratios (ORs) in our study, it remains important to understand the clinical relevance of odds ratios with caution interpreting these findings as relative risk which may overestimate our findings [52]. Further, ORs for technical and clinical success were 1.30 and 2.32 so the odds for EUS-BD were much higher compared to ERCP but the ratios did not reach the level of statistical significance, suggesting this study may be underpowered to make any conclusions. An additional concern with any endoscopic procedure or technique is the learning curve or clinical expertise needed to perform an effective procedure. It is highly possible the technical and clinical success achieved in these studies as well as the rate of AEs may not be generalizable to centers with less familiarity or proven expertise.

Despite these limitations, this study has several strengths. Most importantly, our meta-analysis methodologically summarizes all available data to evaluate feasibility, efficacy, and tolerability of primary EUS-BD. While significant heterogeneity was noted in our meta-analysis and was not surprising given the cumulative nature of reporting results, the prediction interval was calculated to demonstrate and describe the variability, or heterogeneity, of our results within true clinical practice [43]. Although reporting bias of negative studies cannot be ascertained, small case series were eliminated to minimize inherent selection bias associated with them. In addition, this systematic review and meta-analysis evaluates objective data including technical success, clinical success, and AEs of primary EUS-BD, with a subgroup comparison between primary EUS-BD and conventional ERCP. It remains the only meta-analysis to date to evaluate EUS-BD as a primary therapy for distal malignant biliary obstruction. While these procedures are complex and require dedicated devices to which some centers may not have access, our results may also be underappreciated given the need for more experience.

\section{Conclusion}

Overall, this systematic review and meta-analysis found that EUS-BD is a technically feasible, clinically effective, and safe procedure for primary treatment of distal malignant biliary obstruction. When compared to ERCP, EUS-BD had no significant difference in efficacy based on rates of technical and clinical success. In addition, EUS-BD had a lower rate of AEs, most notably lower rates of post-procedure pancreatitis. While the results of this analysis suggest EUS-BD should be increasingly considered as first-line therapy, it is important to note these findings are limited in their ability to translate to endoscopists with limited EUS experience. Furthermore, given the small number of studies in this subset of our analysis, further randomized trials should be performed to identify the ideal patient populations and clinical scenarios in which primary EUS-BD would be most appropriate. 


\section{Competing interests}

Dr. Thompson is a consultant for Boston Scientific, Olympus, and Apollo Endosurgery. Dr. Ryou is a consultant for Medtronic/Covidien, Boston Scientific, Olympus, Fujifilm, GI Windows, EnteraSense, and Pentax.

\section{References}

[1] Speer AG, Cotton PB, Russell RC et al. Randomised trial of endoscopic versus percutaneous stent insertion in malignant obstructive jaundice. Lancet 1987; 2: 57-62

[2] Moss AC, Morris E, Mac Mathuna P. Palliative biliary stents for obstructing pancreatic carcinoma. Cochrane Database Syst Rev 2006: CD004200

[3] Ilnamdar S, Slattery E, Bhalla R et al. Comparison of adverse events for endoscopic vs percutaneous biliary drainage in the treatment of malignant biliary tract obstruction in an inpatient national cohort. JAMA Oncol 2016; 2: $112-117$

[4] Artifon EL, Loureiro JF, Baron TH et al. Surgery or EUS-guided choledochoduodenostomy for malignant distal biliary obstruction after ERCP failure. Endosc Ultrasound 2015; 4: 235-243

[5] Enochsson L, Swahn F, Arnelo U et al. Nationwide, population-based data from 11,074 ERCP procedures from the Swedish Registry for Gallstone Surgery and ERCP. Gastrointest Endosc 2010; 72: 11751184,1184 e1-3

[6] Schofl R. Diagnostic endoscopic retrograde cholangiopancreatography. Endoscopy 2001; 33: $147-157$

[7] KKawakubo K, Kawakami H, Kuwatani M et al. Endoscopic ultrasoundguided choledochoduodenostomy vs. transpapillary stenting for distal biliary obstruction. Endoscopy 2016; 48: 164-169

[8] Lee JH, Krishna SG, Sing A et al. Comparison of the utility of covered metal stents versus uncovered metal stents in the management of malignant biliary strictures in 749 patients. Gastrointest Endosc 2013; 78: $312-324$

[9] Tol JA, van Hooft JE, Timmer R et al. Metal or plastic stents for preoperative biliary drainage in resectable pancreatic cancer. Gut 2016; 65: $1981-1987$

[10] Paik WH, Lee TH, Park DH et al. EUS-guided biliary drainage versus ercp for the primary palliation of malignant biliary obstruction: a multicenter randomized clinical trial. Am J Gastroenterol 2018; 113 : 987-997

[11] Bang JY, Navaneethan U, Hasan M et al. Stent placement by EUS or ERCP for primary biliary decompression in pancreatic cancer: a randomized trial (with videos). Gastrointest Endosc 2018; 88: 9-17

[12] Savader S], Trerotola SO, Merine DS et al. Hemobilia after percutaneous transhepatic biliary drainage: treatment with transcatheter embolotherapy. J Vasc Interv Radiol 1992; 3: 345-352

[13] Sharaiha RZ, Khan MA, Kamal F et al. Efficacy and safety of EUS-guided biliary drainage in comparison with percutaneous biliary drainage when ERCP fails: a systematic review and meta-analysis. Gastrointest Endosc 2017; 85: $904-914$

[14] Giovannini M, Moutardier V, Pesenti C et al. Endoscopic ultrasoundguided bilioduodenal anastomosis: a new technique for biliary drainage. Endoscopy 2001; 33: 898 - 900

[15] Gupta K, Perez-Miranda M, Kahaleh M et al. Endoscopic ultrasoundassisted bile duct access and drainage: Multicenter, long-term analysis of approach, outcomes, and complications of a technique in evolution. J Clin Gastroenterol 2014; 48: 80 - 87
[16] Dhir V, Artifon ELA, Gupta K et al. Multicenter study on endoscopic ultrasound-guided expandable biliary metal stent placement: Choice of access route, direction of stent insertion, and drainage route. Digest Endosc 2014; 26: 430-435

[17] Khashab MA, Kumbhari V, Kalloo AN et al. EUS-guided biliary drainage by using a hepatogastrostomy approach. Gastrointest Endosc 2013; 78: 675

[18] Vila J], Perez-Miranda M, Vazquez-Sequeiros E et al. Initial experience with EUS-guided cholangiopancreatography for biliary and pancreatic duct drainage: a Spanish national survey. Gastrointest Endosc 2012; 76: $1133-1141$

[19] Dhir V, Itoi T, Khashab MA et al. Multicenter comparative evaluation of endoscopic placement of expandable metal stents for malignant distal common bile duct obstruction by ERCP or EUS-guided approach. Gastrointest Endosc 2015; 81: 913 - 923

[20] Park DH, Jang JW, Lee SS et al. EUS-guided biliary drainage with transluminal stenting after failed ERCP: Predictors of adverse events and long-term results. Gastrointest Endosc 2011; 74: 1276 - 1284

[21] Iwashita T, Lee JG, Shinoura S et al. Endoscopic ultrasound-guided rendezvous for biliary access after failed cannulation. Endoscopy 2012; 44: 60-65

[22] Shah JN, Marson F, Weilert F et al. Single-operator, single-session EUSguided anterograde cholangiopancreatography in failed ERCP or inaccessible papilla. Gastrointest Endosc 2012; 75: 56-64

[23] Dhir V, Bhandari S, Bapat M et al. Comparison of EUS-guided rendezvous and precut papillotomy techniques for biliary access (with videos). Gastrointest Endosc 2012; 75: 354 - 359

[24] Attasaranya S, Netinasunton N, Jongboonyanuparp T et al. The Spectrum of endoscopic ultrasound intervention in biliary diseases: a single center's experience in 31 cases. Gastroenterol Res Pract 2012; 2012: 680753

[25] Prachayakul V, Aswakul P. A novel technique for endoscopic ultrasound-guided biliary drainage. World J Gastroenterol 2013; 19: $4758-4763$

[26] Takada J, Carmo AM, Artifon ELA. EUS-guided biliary drainage for malignant biliary obstruction in patients with failed ERCP. J Interventional Gastroenterol 2013; 3: 76-81

[27] Bapaye A, Dubale N, Ahera A. Comparison of endosonography-guided vs. Percutaneous biliary stenting when papilla is inaccessible for ERCP. United Europ Gastroenterol J 2013; 1: 285-293

[28] Kawakubo K, Isayama $\mathrm{H}$, Kato $\mathrm{H}$ et al. Multicenter retrospective study of endoscopic ultrasound-guided biliary drainage for malignant biliary obstruction in Japan. J Hepato-Biliary-Pancreatic Sci 2014; 21: $328-334$

[29] Will U, Fueldner F, Kern C et al. EUS-guided bile duct drainage (EUBD) in 95 patients. Ultraschall Med 2015; 36: 276-283

[30] Weilert F. Prospective evaluation of simplified algorithm for EUSguided intra-hepatic biliary access and anterograde interventions for failed ERCP. Surg Endosc Other Interventional Tech 2014; 28: 3193 3199

[31] Song T], Lee SS, Park DH et al. Preliminary report on a new hybrid metal stent for EUS-guided biliary drainage (with videos). Gastrointest Endosc 2014; 80: 707 - 711

[32] Khashab MA, Valeshabad AK, Afghani E et al. A comparative evaluation of EUS-guided biliary drainage and percutaneous drainage in patients with distal malignant biliary obstruction and failed ERCP. Dig Dis Sci 2015; 60: $557-565$

[33] Dhir V, Bhandari S, Bapat M et al. Comparison of transhepatic and extrahepatic routes for EUS-guided rendezvous procedure for distal CBD obstruction. United Europ Gastroenterol J 2013; 1: 103-108

[34] Khan MA, Akar A, Baron TH et al. Endoscopic ultrasound-guided biliary drainage: a systematic review and meta-analysis. Dig Dis Sci 2016; 61: $684-703$ 
[35] Minaga K, Kitano M. Recent advances in endoscopic ultrasound-guided biliary drainage. Dig Endosc 2018; 30: $38-47$

[36] Liberati A, Altman DG, Tetzlaff J et al. The PRISMA statement for reporting systematic reviews and meta-analyses of studies that evaluate health care interventions: explanation and elaboration. Ann Intern Med 2009; 151: W65 - W94

[37] Stroup DF, Berlin JA, Morton SC et al. Meta-analysis of observational studies in epidemiology: a proposal for reporting. Meta-analysis of observational studies in epidemiology (MOOSE) group. JAMA 2000; 283: $2008-2012$

[38] Higgins JP, Thompson SG, Deeks J] et al. Measuring inconsistency in meta-analyses. BMJ 2003; 327: 557-560

[39] Riley RD, Higgins JP, Deeks JJ. Interpretation of random effects metaanalyses. BMJ 2011; 342: d549

[40] Egger M, Davey SmithG, Schneider M et al. Bias in meta-analysis detected by a simple, graphical test. BMJ 1997; 315: 629-634

[41] Tran A, Villeneuve JP, Bilodeau M et al. Treatment of chronic bleeding from gastric antral vascular ectasia (GAVE) with estrogen-progesterone in cirrhotic patients: an open pilot study. Am J Gastroenterol 1999; 94: 2909-2911

[42] Barbara G, De Giorgio R, Salvioli B et al. Unsuccessful octreotide treatment of the watermelon stomach. J Clin Gastroenterol 1998; 26: $345-346$

[43] Njei B, Laine L. Early Use of TIPS and outcomes patients with cirrhosis acute esophageal variceal bleeding: analysis the U.S. Nationwide Inpatient Sample (NIS) Database, 2000-2010. ACG Annual Scientific Meeting 2018. AJG 2015; 11: 1
[44] Park JK, Woo YS, Noh DH et al. Efficacy of EUS-guided and ERCPguided biliary drainage for malignant biliary obstruction: prospective randomized controlled study. Gastrointest Endosc 2018; 88: 277 282

[45] Nakai $\mathrm{Y}$, Isayama $\mathrm{H}$, Kawakami $\mathrm{H}$ et al. Prospective multicenter study of primary EUS-guided choledochoduodenostomy using a covered metal stent. Endosc Ultrasound 2019; 8: 111-117

[46] Yamao K, Kitano M, Takenaka M et al. Outcomes of endoscopic biliary drainage in pancreatic cancer patients with an indwelling gastroduodenal stent: a multicenter cohort study in West Japan. Gastrointest Endosc 2018; 88: 66 - 75 e2

[47] Hara K, Yamao K, Hijioka S et al. Prospective clinical study of endoscopic ultrasound-guided choledochoduodenostomy with direct metallic stent placement using a forward-viewing echoendoscope. Endoscopy 2013; 45: 392 - 396

[48] Hara K, Yamao K, Niwa Y et al. Prospective clinical study of EUS-guided choledochoduodenostomy for malignant lower biliary tract obstruction. Am J Gastroenterol 2011; 106: 1239-1245

[49] Khan MA, Akbar A, Kamal S et al. Efficacy and safety of endoscopic ultrasound-guided biliary drainage: a systematic review and metaanalysis. Am J Gastroenterol 2015; 110: S5 - S7

[50] Mohan BP, Shakhatreh M, Garg R et al. Efficacy and safety of endoscopic ultrasound-guided choledochoduodenostomy: a systematic review and meta-analysis. J Clin Gastroenterol 2019; 53: 243-250

[51] Freeman ML, Nelson DB, Sherman $S$ et al. Complications of endoscopic biliary sphincterotomy. N Engl J Med 1996; 335: 909-918

[52] Davies HT, Crombie IK, Tavakoli M. When can odds ratios mislead? BMJ 1998; 316: $989-991$ 